\title{
Pyocyanin and 1-Hydroxyphenazine Produced by Pseudomonas aeruginosa Inhibit the Beating of Human Respiratory Cilia In Vitro
}

\author{
Robert Wilson," Tyrone Pitt," Graham Taylor,' David Watson," John MacDermot," \\ David Sykes," David Roberts," and Peter Cole* \\ *Host Defence Unit, Department of Medicine, Cardiothoracic Institute, Brompton Hospital, Fulham Road, London SW3 6 HP; \\ ${ }^{\ddagger}$ Division of Hospital Infection, Central Public Health Laboratory, London NW9 5HT; and \$ Department of Clinical Pharmacology, \\ Royal Postgraduate Medical School, Hammersmith Hospital, London W12 OHS, England
}

\begin{abstract}
Pseudomonas aeruginosa culture filtrates varied in their ability to slow human ciliary beat frequency (7-71\%). This activity did not correlate with known virulence factors. However, a close correlation $(r=0.97)$ existed between ciliary slowing and pigment content. In a prolonged culture, the increase in activity correlated $(r=0.94)$ with pigment accumulation. Gel filtration of lyophilized filtrate yielded a single peak of activity corresponding to the pigment fraction. Pyocyanin extracted from an active strain, and 1-hydroxyphenazine were purified by high performance liquid chromatography, and characterized by ultraviolet absorbance spectra and mass spectrometry. Both slowed cilia in a dose-dependent manner, and were synthesized and shown to be indistinguishable from the biological compounds. Pyocyanin caused gradual onset of slowing and ultimate widespread ciliostasis with epithelial disruption. 1-hydroxyphenazine caused rapid onset of ciliary slowing associated with dyskinesia and ciliostasis. Pyocyanin assayed within filtrates accounted for a significant proportion of the bioactivity present.
\end{abstract}

\section{Introduction}

The lungs of patients with cystic fibrosis and other causes of severe bronchiectasis are frequently colonized by Pseudomonas aeruginosa. This leads to clinical deterioration, worsening lung function, and frequently to death (1). Mucociliary clearance is the first-line defense mechanism of the human respiratory tract against inhaled particles including bacteria. Defective mucociliary activity is associated with recurrent chest infection and finally bronchiectasis (2). We have previously reported the phenomenon of slowing of human nasal ciliary beat frequency in vitro by $P$. aeruginosa culture filtrates (3). We report here the extraction, purification, and characterization of two pseudomonas factors associated with this activity.

\section{Methods}

\section{Preparation and assay of bacterial culture filtrates}

(a) Overnight aerobic incubation of sputum at $37^{\circ} \mathrm{C}$ on plates containing cetrimide nalidixic acid agar (Oxoid Ltd., Basingstoke, England) yielded 17 isolates of $P$. aeruginosa from patients with bronchiectasis (six with cystic fibrosis: isolates $3,4,7,12,14$, and 16 ). The purity of isolates was

Address correspondence to Dr. Wilson, Host Defence Unit, Department of Medicine, Cardiothoracic Institute, Brompton Hospital, Fulham Road, London, SW3 6HP, England. 1986.

Received for publication 26 August 1985 and in revised form 15 July

J. Clin. Invest.

(c) The American Society for Clinical Investigation, Inc.

$0021-9738 / 87 / 01 / 0221 / 09 \quad \$ 1.00$

Volume 79, January 1987, 221-229 confirmed by subculture and overnight incubation. All experiments were performed with fresh isolates. After incubation in $20 \mathrm{ml}$ of medium 199 (with Earle's salts and Hepes; Flow Laboratories, Inc., McLean, VA) at $37^{\circ} \mathrm{C}$ for $18 \mathrm{~h}$ and a viable count, the cultures were centrifuged at 4,656 $g$ for $30 \mathrm{~min}$ at $4^{\circ} \mathrm{C}$. The supernatant fluids were filtered $(0.2 \mu \mathrm{m}$ Acrodisc; Gelman Sciences, Inc., Ann Arbor, MI) to yield bacteria-free filtrates (pH 7.0-7.3; sterility of aliquots tested overnight at $37^{\circ} \mathrm{C}$ ).

(b) Strips of normal human nasal ciliated epithelium obtained from the inferior turbinate using a cytology brush (4) were dispersed in medium 199 , divided into two equal samples, and centrifuged at $186 \mathrm{~g}$ for 10 min. The supernatant medium 199 was aspirated and replaced by an equal volume of fresh medium 199 in one sample (control) and by the pseudomonas filtrate in the other (test). A sealed microscope coverslipslide was prepared for measurement of ciliary beat frequency $(\mathrm{CBF})^{1}$ by a photometric technique $(3,4)$. The microscope coverslip-slide preparation was placed on an electronically controlled warmstage (Microtec, Oxford, England) at $37^{\circ} \mathrm{C}$ and mounted on a Leitz Dialux 20 phasecontrast microscope. A Leitz MPV compact microscope photometer transduced light intensity into an electrical signal. Strips of epithelium with beating cilia were viewed directly at a magnification of 320 by bright field illumination. The cilia were positioned to interrupt the passage of light through a small diaphragm into the photometer, and the electrical signal generated was converted into a reading of CBF (in hertz) (5). Direct viewing of the cilia allowed an assessment of their beating pattern. Ciliary dyskinesia was defined as absence of the usual coordinated ciliary movement, i.e., cilia may be seen to perform vibration about a vertical axis and those on the same strip may beat in opposite directions. Ciliostasis was defined as complete absence of ciliary movement.

CBF was measured at 30-min intervals for $4 \mathrm{~h}$ after initial equilibration at $37^{\circ} \mathrm{C}$ over $10 \mathrm{~min}$. Six or more strips of ciliated epithelium were identified on the slide and the latter marked for reidentification at subsequent time points. These six strips were all used as sites of 10 subsequent $\mathrm{CBF}$ readings at each time point. The mean of these 10 readings at each time point was calculated for both control and test slides. Ciliary dyskinesia or ciliostasis was noted. Because static cilia become progressively more difficult to distinguish from unciliated epithelium, the CBF of static cilia was not counted unless there were insufficient groups of beating cilia to allow 10 separate readings of $\mathrm{CBF}$ to be made. This methodology underestimates, therefore, the overall ciliary-slowing activity of the bacterial preparations.

(c) The colony type of each isolate was noted after overnight culture on blood agar. Protease activity of the filtrate was estimated spectrophotometrically on two separate days by gentle rotation of $5 \mathrm{ml}$ of filtrate with $10 \mathrm{mg}$ of azocaesin (Sigma Chemical Co., St. Louis, MO) at $37^{\circ} \mathrm{C}$ for $60 \mathrm{~min}$, then precipitation of excess azocaesin with $250 \mu 1100 \%$ TCA. After separation from the precipitate, the absorbance of the now orange filtrate was read on an ultraviolet (UV) spectrophotometer at 340 $\mathrm{nm}$, the result adjusted to a standard viable count of $5 \times 10^{8}$ organisms and compared with results obtained after similar incubation with porcine trypsin (Sigma Chemical Co.). The result was presented as trypsin equivalent per $5 \times 10^{8}$ organisms. Specific elastase activity of the filtrate was estimated spectrophotometrically on two occasions after gentle rotation

1. Abbreviations used in this paper: 1-hp, 1-hydroxyphenazine; 1-mp, 1methoxyphenazine; $\mathrm{CBF}$, ciliary beat frequency; d.e.i., desorption electron impact; GC, gas chromatography; LC, liquid chromatography; MS, mass spectrometry; TFA, trifluoroacetic acid. 
of $5 \mathrm{ml}$ of filtrate with $20 \mathrm{mg}$ of congo-red elastin (Sigma Chemical Co.) for $18 \mathrm{~h}$ at $37^{\circ} \mathrm{C}$. Haemolytic activity of the filtrate was estimated by incubation with $5 \%$ washed sheep red blood cells for $3 \mathrm{~h}$ at $37^{\circ} \mathrm{C}$. Production of other enzymes by the isolates was estimated by agar plate techniques in which substrate was incorporated into an agar base with appropriate nutrient; lipase (6), DNAse (7), or lecithinase (8). The absorbance of each filtrate was measured at $400 \mathrm{~nm}$ to determine pigment content.

(d) Lipopolysaccharide was prepared by the method of Chester, Meadow, and Pitt (9). Alginate from a mucoid strain of $P$. aeruginosa was prepared by the method of Pitt and Raisbeck (10), then dried in vacuo after deproteinization with butanol and chloroform. Each was dissolved in medium $199(1 \mathrm{mg} / \mathrm{ml})$ and tested for inhibition of CBF. The activity of filtrates of two strains, PA 103 (high exotoxin A, nonprotease producer) and PA 8505 (nonproducer of exotoxin A and protease), was also assayed for inhibition of CBF. The activity of five filtrates from nonmucoid strains was compared with the activity of five filtrates from mucoid strains.

(e) Sequential samples of supernatant fluid taken during a continuous 4-d culture of isolate 4 in medium 199 at $37^{\circ} \mathrm{C}$ were centrifuged $(4,656$ g; $30 \mathrm{~min})$ then filtered ( $0.2 \mu \mathrm{m}$ Acrodisc; Gelman Sciences, Inc.). The spectrophotometric absorbance of each filtrate was measured at $400 \mathrm{~nm}$ and its activity assayed for inhibition of CBF. The filtrate of a 4-d culture of Staphylococcus aureus in medium 199 at $37^{\circ} \mathrm{C}$ was also assayed for ciliary-slowing activity.

\section{Gel filtration}

Three isolates (Nos. 2, 5, and 8) were separately cultured in $100 \mathrm{ml}$ of medium $i 99$ for $18 \mathrm{~h}$ at $37^{\circ} \mathrm{C}$, and the cultures centrifuged and filtered $(0.2 \mu \mathrm{m}$ Acrodisc; Gelman Sciences, Inc.) to yield bacteria-free preparations, which were immediately frozen and then lyophilized overnight. $100 \mathrm{ml}$ of medium 199 were treated similarly to act as control. Each solid was reconstituted in $5 \mathrm{ml}$ distilled water, $2 \mathrm{ml}$ of this being passed down a Sephacryl-200 gel filtration column (Pharmacia Fine Chemicals, Piscataway, NJ) at $4^{\circ} \mathrm{C}$.

Phosphate-buffered saline (PBS) was used to elute, and 4-ml aliquots were collected and stored at $-30^{\circ} \mathrm{C}$ until their cilioinhibitory activity was tested. The column void volume was $60 \mathrm{ml}$, and absorbance at 280 $\mathrm{nm}$ indicated that pseudomonas products appeared between elution volumes of 92 and $148 \mathrm{ml}$. Cilia beat normally in PBS and aliquots from the column were tested for their cilioinhibitory activity against a control of PBS alone. Osmolality of aliquots was checked by absence of crenation of human red blood cells and corrected with deionized water if required. The percent ciliary slowing produced by each aliquot was calculated as 100 minus (minimum CBF during experiment/control CBF at same experimental time-point $\times 100$ ). The absorbance of each aliquot was measured at $400 \mathrm{~nm}$.

\section{Factor purification, synthesis, and assay}

Pyocyanin and 1-hydroxyphenazine (1-hp) were prepared by methods adapted from those described by Armstrong et al. (11).

\section{PREPARATION OF PYOCYANIN}

10 King's A Agar (12) plates were cultured aerobically at $37^{\circ} \mathrm{C}$ for 18 $\mathrm{h}$, left at room temperature for a further $24 \mathrm{~h}$, the organisms washed from the surface of the agar using sterile distilled water, and the agar cut aseptically into $1-\mathrm{cm}$ squares that were placed in a sterile glass bottle. A 20-ml aliquot of dry chloroform was added, the mixture shaken vigorously and the chloroform (colored blue) removed by pipette and filtered (No. 1, double; Whatman Chemical Separation, Inc., Clinton, NJ). The procedure was repeated to extract the remaining pigment. The chloroform was evaporated in an air stream and the blue sediment redissolved in 5 $\mathrm{ml}$ of dry chloroform. Dry petroleum ether was added dropwise until blue crystals of pyocyanin formed $(\sim 15 \mathrm{ml})$. The apple-green supernatant was decanted and the blue crystals recrystallized from chloroform. The crystals were dissolved in distilled water, filtered $(0.2 \mu \mathrm{m}$ Acrodisc; Gelman Sciences, Inc.), and freeze-dried for estimation of weight. $2 \mathrm{mg}$ of crude crystalline pyocyanin was obtained.

\section{PREPARATION OF 1-HP BY BASE HYDROLYSIS}

An alkaline solution $(0.2 \mathrm{~N} \mathrm{NaOH})$ of pyocyanin was left at room temperature for $18 \mathrm{~h}$, during which time the color changed from blue to red-violet. Acidification with $1 \mathrm{~N} \mathrm{HCl}$ yielded a yellow precipitate of crude 1-hp, which was dissolved in distilled water and freeze-dried for weight estimation. $200 \mu \mathrm{g}$ of crude 1-hp was obtained from $4 \mathrm{mg}$ of pyocyanin.

\section{SYNTHESIS OF 1-HP AND PYOCYANIN}

All chemicals were obtained from Aldrich Chemical Co. (Milwaukee, WI) unless otherwise stated. HPLC (high pressure liquid chromatography) solvents were purchased from Rathburn, Walkerburn, England; water was of Milli Q quality.

1-hp and pyocyanin were synthesized following the method of Armstrong et al. (11). Essentially, 1-methoxy catechol $(2 \mathrm{~g})$ was oxidized with lead dioxide $(40 \mathrm{~g})$ in dry benzene, and the resultant quinone coupled with o-phenylenediamine (1.8 g) forming 1-methoxyphenazine (1-mp). $1-\mathrm{mp}$ was crystallized from pyridine/water to give red-orange crystals in $12.5 \%$ yield. 1-mp was hydrolyzed in $60 \% \mathrm{HBr}(5 \mathrm{~h}$, reflux), neutralized, and extracted into ether to form 1-hp in good yield. 1-hp was purified by exhaustive ether extraction, first from base $(10 \% \mathrm{NaOH})$ and then from acid ( $5 \%$ acetic acid, $\mathrm{pH} 3$ ).

1-hp was converted into pyocyanin by heating with methyl sulphate $\left(100^{\circ} \mathrm{C}, 10 \mathrm{~min}\right)$. Crude pyocyanin was precipitated by addition of dry ether. The solid was dissolved in water (pH 11) and extracted into chloroform. Pyocyanin was further extracted into $5 \% \mathrm{HCl}$, and back into chloroform after neutralization. Excess solvent was removed under nitrogen to yield a dark blue solid.

\section{PURIFICATION AND CHARACTERIZATION}

Biological and synthetic 1-hp and pyocyanin were purified by HPLC before biological or structural studies.

HPLC. HPLC was undertaken on a Waters gradient elution system, monitoring at 280 and $254 \mathrm{~nm}$ in the UV. Elution was carried out either:

(i) at $2 \mathrm{ml} / \mathrm{min}$ on a Nova Pak octadecylsilyl column (Waters Associates, Millipore Corp., Milford, MA) with acetonitrile/water/trifluoroacetic acid (TFA) (100:0:0.04 vol/vol/vol) isocratically for $5 \mathrm{~min}$ followed by a 10-min linear gradient to acetonitrile/water/TFA (60:40:0.04 vol/vol/vol).

(ii) For thermospray liquid chromatography/mass spectrometry (LC/ MS), at $1.8 \mathrm{ml} / \mathrm{min}$ on a Nova Pak octadecylsilyl column (Waters Associates, Millipore Corp.) eluting with a 10-min linear gradient from aqueous ammonium acetate $(0.05 \mathrm{M})$ to $70 \%$ acetonitrile/water-containing $0.05 \mathrm{M}$ ammonium acetate.

(iii) For assay of pyocyanin in culture filtrates, at $2 \mathrm{ml} / \mathrm{min}$ on a $\mu$ Bondapak C18 column $(30 \times 0.8 \mathrm{~cm}$; Waters Associates, Millipore Corp.) with a 20 -min linear gradient from 0 to $40 \%$ propan-2-ol in aqueous acetic acid (5\%).

$M S$. Mass spectra were recorded in the electron impact (e.i.), desorption e.i. (d.e.i.), or thermospray ion modes (where applicable) on a mass spectrometer (model 4,500; Finnigan, Sunnyvale, CA). Samples were introduced either directly, or via gas chromatography (GC)/HPLC. The HPLC was coupled to the mass spectrometer via a "Vestal" thermospray interface (Finnigan, Sunnyvale, CA). The source block was kept at $200^{\circ} \mathrm{C}$ and the interface jet was operated at $140^{\circ} \mathrm{C}$. HPLC/MS was undertaken with solvent system ii, although for loop injection of pyocyanin, the salt concentration was varied.

GC. GC was undertaken on an SE54 capillary column with helium carrier gas. Samples in octane were injected in the splitless mode at $250^{\circ} \mathrm{C}$ injector temperature. The injector temperature was also varied between $200^{\circ}-300^{\circ} \mathrm{C}$ for pyocyanin. Elution was carried out for $1 \mathrm{~min}$ isothermally $\left(100^{\circ} \mathrm{C}\right)$ followed by a linear gradient of $20^{\circ} \mathrm{C} / \mathrm{min}$ to $300^{\circ} \mathrm{C}$. The GC was monitored directly in the mass spectrometer in the e.i. mode.

UV spectrophotometry. Full UV spectra were obtained in methanol, $0.1 \mathrm{M} \mathrm{HCl}$, or $0.1 \mathrm{M} \mathrm{NaOH}$ on a spectrophotometer (model 555; PerkinElmer Corp., Norwalk, CT). Quantitation for bioassay was based on the 
absorbance in $0.1 \mathrm{M} \mathrm{HCl} /$ pyocyanin: $\lambda \max 278 \epsilon \max 50,000$; and 1hp: $\lambda \max 273 \mathrm{~nm}, \epsilon \max 30,000$.

\section{ASSAY OF CRUDE AND PURIFIED FACTORS ON CBF}

The crude and purified (HPLC) biological and the synthetic pyocyanin and 1-hp were dissolved in PBS for assay of their cilioinhibitory activity.

\section{EFFECT OF ADJUSTING pH}

In preliminary experiments, the effect of $\mathrm{pH}$ on $\mathrm{CBF}$ was tested in PBS for the range 6.5 to 8.0. The CBF assay was then performed after adjusting the $\mathrm{pH}$ (with $1 \% \mathrm{HCl}$ or $\mathrm{NaOH}$ ) of the bacterial filtrates, and of the purified synthetic 1-hp and pyocyanin. In each experiment the $\mathrm{pH}$ of the control slide was identical to that of the test slide.

\section{Assay of cilioinhibitory factors within culture filtrates}

After characterization and synthesis of pyocyanin and 1-hp, methodology was developed to assay for the presence or absence of these compounds in culture filtrates without prior extraction. Nine new isolates (Nos. 917) were used to produce 18-h culture filtrates for assay of ciliary-slowing activity. Two longer cultures (a 48-h culture of one of the new isolates [No. 13] and a sample from the 4-d culture of isolate No. 4 described earlier) were also similarly assayed. Pyocyanin and 1-hp were chromatographed on a $\mu$ Bondapak C18 HPLC column in system iii (13). The HPLC eluate was monitored at 280 and $254 \mathrm{~nm}$. Pyocyanin eluted as an $A_{280} \mathrm{UV}$-absorbing peak at $25.4 \mathrm{ml}$ (and 1-hp at $50 \mathrm{ml}$ ). The peak height $\left(\mathrm{A}_{280}\right)$ was proportional to the amount of pyocyanin loaded, allowing a standard curve to be constructed for synthetic pyocyanin across the range $50-500 \mathrm{ng}$. Culture filtrates (250 $\mu \mathrm{l}$ in medium 199) were similarly chromatographed, and $\mathrm{A}_{280}$ peak height for pyocyanin and 1hp measured.

The remaining filtrate was made basic (pH 12) with $5 \% \mathrm{NaOH}$ and extracted twice with two volumes of chloroform. Chloroform was removed under a stream of nitrogen and the full UV spectrum obtained in $0.1 \mathrm{M} \mathrm{HCl}$.

\section{Statistical analysis}

Each experiment yielded a series of $10 \mathrm{CBF}$ readings at each timepoint, and the mean $C B F$ at each time point was calculated for test and control preparations. The slowest mean CBF of the test preparation was identified and compared with the control mean at the same timepoint by the unpaired $t$ test (20 readings, 18 d.f.).

\section{Results}

Culture filtrates. The slowing of human nasal ciliary beating by 17 filtrates of medium from 18-h cultures of separate isolates of $P$. aeruginosa was measured. Three patterns of ciliary inhibition were observed: inhibition with no recovery during the 4-h experiment-by the filtrates from seven isolates (Nos. 1, 2, and 9-13); inhibition with recovery-by the filtrates from six isolates (Nos. 3-7, 14); and no inhibition at all-by the filtrates from four isolates $(8,15-17)$. For clarity these patterns are illustrated in Fig. 1 by 10 of these 17 isolates. The CBF of control specimens did not vary significantly over $4 \mathrm{~h}$ (Fig. 1). Marked slowing was associated with dyskinesia, which usually preceded ciliary stasis in some areas of the epithelium and disruption of the epithelium itself. Complete reversal of the filtrate effect could be demonstrated by washing the cilia with fresh culture medium before ciliostasis and disruption were observed.

This activity of the filtrates neither correlated with their protease, specific elastase or haemolytic activity, nor with production of other pseudomonas enzymes as detected by plate techniques (Table I), nor with other virulence factors as demonstrated in experiments using lipopolysaccharide, alginate, and specific strains (Table II). Five nonmucoid strains produced more active filtrates than five mucoid strains (mean $46 \%$ ciliary slowing compared with $12 \%$ ). UV absorbance at $400 \mathrm{~nm}$ indicated a close correlation $(r=0.97)$ between percent ciliary slowing and pigment contained in the filtrate (Table I).

Sequential samples of supernatant fluid taken during a continuous 4-d culture of isolate 4 in medium 199 at $37^{\circ} \mathrm{C}$ increased progressively in their capacity to slow ciliary beating when compared with control medium alone (Table III). There was close correlation $(r=0.94)$ between this activity and the gradually increasing pigment accumulation in the filtrate. After a 4-d culture of $S$. aureus in medium 199 at $37^{\circ} \mathrm{C}$ to control for medium exhaustion, a filtrate of the culture medium did not slow ciliary beating. $S$. aureus has previously been shown not to produce factors slowing ciliary beating in vitro (3). Therefore, it is unlikely that the activity of the filtrates of $P$. aeruginosa was due to exhaustion of the medium 199 of factors necessary for normal ciliary beating.

Gel filtration. Gel filtration of the filtrates (lyophilized and later reconstituted in water) obtained from strongly cilioinhibitory strain 2 and protease-producing strain 5 yielded fractions with a single peak of activity (Fig. 2) corresponding to the fractions containing pigment. Fractions from similarly treated control medium and from noncilioinhibitory strain 8 were without activity.

Characterization of 1-hp and pyocyanin. Authentic 1-hp and pyocyanin were synthesized. GC/MS and thermospray LC/MS methodologies were developed and applied both to define the molecular weight and structure of the synthetic materials, and also to facilitate gas chromatographic/mass spectrometric comparison of the synthetic and natural species. The natural and synthetic compounds were indistinguishable by all spectroscopic, mass spectrometric, and chromatographic tests.

Synthetic 1-hp was characterized in terms of its UV absorbance spectrum and e.i. mass spectrum. The full UV spectrum of 1 -hp in $0.1 \mathrm{M} \mathrm{HCl}$ showed an intense absorbance at $\lambda$ max $=273 \mathrm{~nm}(\epsilon=30,000)$ and a series of weaker peaks at $\lambda=363$, 370 , and $383 \mathrm{~nm}(\epsilon=5,500,6,000$, and 8,500 , respectively). In $0.1 \mathrm{M} \mathrm{NaOH}$, the $\lambda$ max shifted from 273 to $291 \mathrm{~nm}$ (14). 1hp chromatographed on GC with a retention time of $7.2 \mathrm{~min}$, and on HPLC at 12.6 or $10.6 \mathrm{~min}$ (systems i and ii). The HPLC/ MS thermospray mass spectrum showed an intense molecular ion species $(\mathrm{M}+\mathrm{H})^{+}$at $\mathrm{m} / \mathrm{z} 197$ defining the molecular weight as 196. The e.i. mass spectrum of 1-hp (post-GC) showed two intense ions at $\mathrm{m} / \mathrm{z} 196\left[\mathrm{M}^{+}\right]$and $168\left[\mathrm{M}^{+}-\mathrm{CO}\right]$ together with minor ions at $\mathrm{m} / \mathrm{z} 154,157,142,140,129,122,115,114,102$, 98 , and 77 . The spectrum was in good agreement with that reported for the isomer 2-hp (15).

Natural 1-hp cochromatographed with synthetic material both on GC and HPLC. The UV and mass spectra (e.i. and thermospray) were indistinguishable from those reported above for synthetic 1-hp.

Synthetic pyocyanin chromatographed on HPLC with retention times of 10.4 or $7 \mathrm{~min}$ (systems $\mathrm{i}$ and ii). It possesses a characteristic UV spectrum in both methanol $(\lambda 1=238, \epsilon 1$ $=26,840, \lambda 2=316 \mathrm{~nm}, \epsilon 2=30,000, \lambda 3=690$ [broad], and $\epsilon 3$ $=4,210)$ and in $0.1 \mathrm{M} \mathrm{HCl}(\lambda 1=278, \epsilon 1=50,000, \lambda 2=385$, $\epsilon 2=21,840, \lambda 3=520$ [broad], and $\epsilon 3=3,160$ ). Under normal thermospray HPLC conditions $(0.05 \mathrm{M}$ ammonium acetate in the solvents), mass spectrometric ionization yielded an intense ion species at $\mathrm{m} / \mathrm{z} 212$, with a weaker ion at $\mathrm{m} / \mathrm{z} 211$ (corresponding to $\mathrm{M}+\mathrm{H}^{+}$). At low ammonium ion concentrations, the intensity of $\mathrm{m} / \mathrm{z} 211$ increased until it became the major ion. The d.e.i. probe mass spectra showed the presence of both the protonated $\left(\mathrm{M}+\mathrm{H}^{+}\right)$species $(\mathrm{m} / \mathrm{z} 211,196,168,140,129)$ 


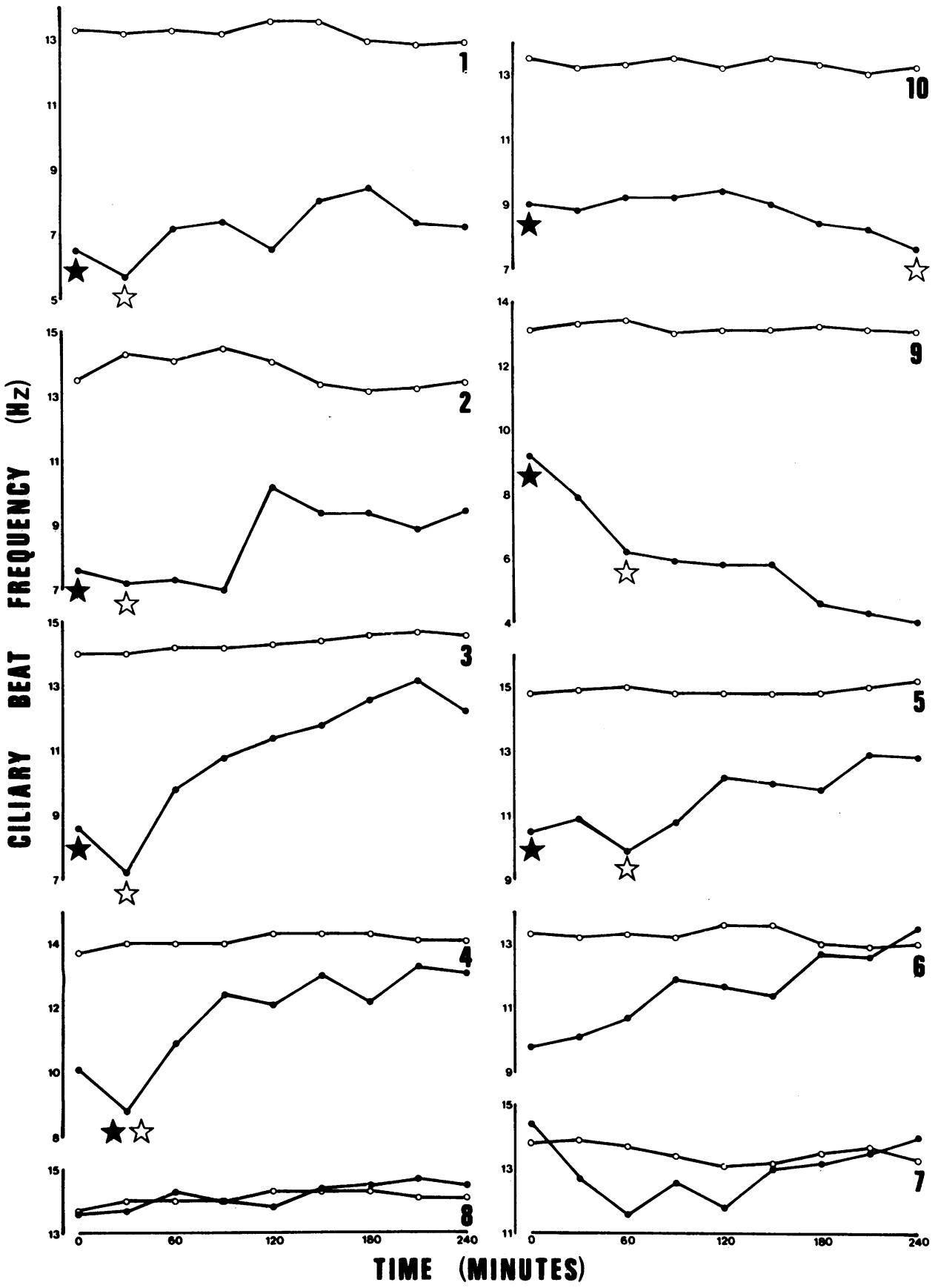

Figure 1. The effect of culture filtrates from ten $P$. aeruginosa isolates on human ciliary beat frequency (CBF) in vitro. The CBF produced by each culture filtrate (solid circles) and the time-based control CBF (open circles) are shown. The top right hand corner of each graph shows the isolate reference number. Filled stars, ciliary dyskinesia; open stars, ciliostasis. and deprotonated $\left(\mathrm{M}^{+}\right)$species: $\mathrm{m} / \mathrm{z} 224\left(\mathrm{M}^{+}+\mathrm{CH}_{2}\right.$, [transmethylation (11)], $\left.210\left(\mathrm{M}^{+}\right), 196\left(\mathrm{M}^{+}-\mathrm{CH}_{3}{ }^{\circ}\right), 181,168,129\right)-$ as expected, the protonated species desorbed from the probe at lower current. A sample of pyocyanin injected onto the gas chromatograph at $250^{\circ} \mathrm{C}$ eluted as a single peak with retention time $7.2 \mathrm{~min}$. The e.i. spectrum was indistinguishable from 1hp. At higher injector temperatures, a species cochromatographing with 1-mp was also observed (retention time $8.1 \mathrm{~min}$ ); the e.i. MS spectrum (m/z $210\left(\mathrm{M}^{+}\right), 209,181,180,179,167$, $153,140,127,114$, and 102) was similar, both to that obtained with our synthetic 1-mp and that reported by Holliman et al. (15).

Natural pyocyanin cochromatographed on HPLC with synthetic material and was indistinguishable from it in terms of its
UV and mass spectra (under both d.e.i. and thermospray conditions).

Bioassay of crude pyocyanin and 1-hp. Crude pyocyanin dissolved in PBS $(16 \mu \mathrm{g} / \mathrm{ml})$ caused ciliostasis and epithelial disruption during the 4-h experiment. At $4 \mu \mathrm{g} / \mathrm{ml}$, gradual ciliary slowing occurred and at $4 \mathrm{~h}$ caused a test value of $9.3 \mathrm{~Hz}$, and a control value of $14.2 \mathrm{~Hz}$. Crude 1-hp dissolved in PBS caused early onset of ciliary slowing with dyskinesia and ciliostasis (32 $\mu \mathrm{g} / \mathrm{ml}$ ), and at $8 \mu \mathrm{g} / \mathrm{ml}$ caused a test value of $11.4 \mathrm{~Hz}$, and a control value of $13.0 \mathrm{~Hz}$ after $60 \mathrm{~min}$.

Bioassay of purified factor. Pyocyanin extracted from an active strain and its base hydrolysis product, 1-hp, were purified by reverse-phase HPLC. HPLC of the apple-green supernatant remaining after pyocyanin crystallization demonstrated the 
Table I. Correlation of Colony Type, Pigment Production, and Enzymic Properties of P. aeruginosa with Their Cilioinhibitory Properties

\begin{tabular}{|c|c|c|c|c|c|c|c|c|c|}
\hline Isolate & Colony type & $\begin{array}{l}\text { Filtrate } \\
\text { absorbance }\end{array}$ & Slowing of CBF & Filtrate protease & $\begin{array}{l}\text { Filtrate elastase } \\
\text { activity }\end{array}$ & $\begin{array}{l}\text { Filtrate hemolytic } \\
\text { activity }\end{array}$ & $\begin{array}{l}\text { Lipase } \\
\text { activity }\end{array}$ & $\begin{array}{l}\text { Lecithinase } \\
\text { activity }\end{array}$ & $\begin{array}{l}\text { DNAse } \\
\text { activity }\end{array}$ \\
\hline & & $400 \mathrm{~nm}^{*}$ & $\%(S D \text { in } H z)^{*}$ & $\begin{array}{l}\mu \mathrm{g} \text { trypsin equivalent to } \\
5 \times 10^{8} \text { organisms }\end{array}$ & & & & & \\
\hline 1 & Nonmucoid & 0.338 & $57(1.8)^{8}$ & 5.2 & $1+$ & 0 & $1+$ & $1+$ & $3+$ \\
\hline 2 & Nonmucoid & 0.288 & $51(2.0)^{8}$ & 14.8 & $2+$ & 0 & $3+$ & $3+$ & 0 \\
\hline 3 & Nonmucoid & 0.230 & $49(2.2)^{8}$ & 1.1 & 0 & 0 & $3+$ & $1+$ & 0 \\
\hline 4 & Nonmucoid & 0.240 & $37(1.4)^{8}$ & 7.7 & 0 & 0 & $2+$ & $1+$ & 0 \\
\hline 5 & Nonmucoid & 0.224 & $34(3.0)^{8}$ & 37.5 & $3+$ & 0 & $3+$ & $2+$ & $1+$ \\
\hline 6 & Nonmucoid & 0.156 & $26(1.5)^{5}$ & 1.7 & 0 & 0 & $3+$ & 0 & 0 \\
\hline 7 & Mucoid & 0.116 & $15(1.8)^{8}$ & 0.0 & 0 & 0 & $3+$ & $3+$ & $3+$ \\
\hline 8 & Mucoid & 0.014 & $3(1.0)^{\S}$ & 0.0 & 0 & 0 & $3+$ & $3+$ & 0 \\
\hline
\end{tabular}

* Correlation coefficient between filtrate absorbance and percent ciliary slowing $=0.97$. $\$$ ciliary slowing $=100$

$-\left[\frac{\text { minimum CBF during experiment }}{\text { control CBF at same experimental time point }} \times 100\right] .{ }^{s} P<0.001 t$ test, test vs. control at same experimental point, 18 d.f.

presence of both pyocyanin and 1-hp. Pyocyanin and 1-hp were characterized in terms of UV absorbance spectra and MS (molecular weights 210 and 196 respectively).

Pyocyanin dissolved in PBS produced gradual slowing of CBF without recovery (Figs. 3 and 4). Ciliary dyskinesia was observed only late in the experiments when ciliostasis and epithelial disruption were also noted. In control experiments using PBS alone, CBF did not vary significantly over 4 h (Fig. 3), however over $36 \mathrm{~h} \mathrm{CBF}$ was more variable (Fig. 4). Significant slowing $(P<0.001, t$ test) occurred at $20 \mu \mathrm{M}$ at $30 \mathrm{~min}$ (Fig. 3), at $1 \mu \mathrm{M}$ at $20 \mathrm{~h}$ and at $0.1 \mu \mathrm{M}$ at $36 \mathrm{~h}$ (Fig. 4). In contrast, Fig. 3 shows 1 -hp to produce rapid onset of ciliary slowing, dyskinesia, and some immediate ciliostasis but some recovery of beating during the course of the experiment (the pattern seen with some isolates in Fig. 1). However, significant changes $(P$ $<0.001$ ) only occurred at a concentration of $10 \mu \mathrm{M} 1$-hp (Fig. 3) even with more prolonged exposure (Fig. 4). The activity of pyocyanin dissolved in PBS did not change after incubation at $37^{\circ} \mathrm{C}$ for $4 \mathrm{~h}$; similarly, its UV spectrum and HPLC retention time were unchanged after $4 \mathrm{~h}$ in $0.04 \%$ TFA or methanol.

Synthetic 1-hp and pyocyanin were dissolved in PBS and assayed as above. Identical patterns of ciliary slowing were produced, and equivalent concentrations gave similar quantitative CBF results compared with control: $50 \mu \mathrm{M} 1-\mathrm{hp}$, a test value of $7.8 \mathrm{~Hz}$, and a control value of $12.8 \mathrm{~Hz}(60 \mathrm{~min})$ with widespread

Table II. Effect on CBF of Pseudomonas

Lipopolysaccharide, Alginate, and 18-h Culture

Filtrates $(0.2 \mu \mathrm{m})$ of Strains PA103 and PA8505

\begin{tabular}{llll}
\hline & $\begin{array}{l}\text { Minimum CBF } \\
\text { during 4 } \\
\text { of exposure }\end{array}$ & & $\begin{array}{l}\text { Control CBF at } \\
\text { same experimental } \\
\text { time point }\end{array}$ \\
\hline & $H z(S D)$ & & $H z(S D)$ \\
Lipopolysaccharide & & & \\
$\quad(1 \mathrm{mg} / \mathrm{ml})$ & $12.9(1.2)$ & NS & $13.7(1.6)$ \\
Alginate $(1 \mathrm{mg} / \mathrm{ml})$ & $12.8(1.0)$ & NS & $13.3(0.6)$ \\
PA 103 & $12.4(1.1)$ & NS & $12.6(1.4)$ \\
PA 8505 & $5.6(1.3)$ & $P<0.001$ & $12.8(1.1)$ \\
\hline
\end{tabular}

$P<0.001, t$ test, 18 d.f. dyskinesia and ciliostasis; $10 \mu \mathrm{M} 1-\mathrm{hp}$, a test value of $10.2 \mathrm{~Hz}$, and a control value of $12.8 \mathrm{~Hz}(60 \mathrm{~min})$ with dyskinesia only. $75 \mu \mathrm{M}$ pyocyanin, a test value of $6.8 \mathrm{~Hz}$, and a control value of $11.9 \mathrm{~Hz}$ (240 min) with widespread epithelial disruption and ciliostasis; $20 \mu \mathrm{M}$, a test value of $9.1 \mathrm{~Hz}$, and a control value of $11.9 \mathrm{~Hz}$ (240 $\mathrm{min}$ ) with some epithelial disruption and ciliostasis.

Effect of $\mathrm{pH}$. In the preliminary experiments CBF did not vary significantly (unpaired $t$ test, 18 d.f.) within the $\mathrm{pH}$ range 7.0 to 8.0. When CBF measured within the range 6.5 to 7.0 was compared with that measured within the range 7.0 to 8.0 , a reduction in CBF of $<10 \%$ was noted. This is in agreement with previously published data (16). Subsequent experiments were controlled with slides at identical $\mathrm{pH}$ to the test slide. Increasing the $\mathrm{pH}$ of the bacterial filtrates from 6.5 to 8.0 markedly reduced their cilioinhibitory properties (at pH 6.5, 43\% max ciliary slow-

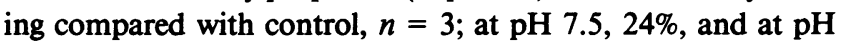
8.0, 5\%). A pH change from 7.5 (used in the above experiments with biological and synthetic $1-\mathrm{hp}$ ) to 6.5 profoundly increased the cilioinhibitory properties of synthetic 1-hp and synthetic pyocyanin (compared with control medium pH 6.5). At pH 6.5,

Table III. Effect of Centrifuged, Filtered $(0.2 \mu \mathrm{m})$ Aliquots Removed from a 4-d Culture of P. aeruginosa (Strain 4) in Medium 199 on the Beat Frequency of Human Nasal Cilia in Vitro

\begin{tabular}{llll}
\hline & & \multicolumn{2}{l}{$\begin{array}{l}\text { Minimum CBF during 4 h } \\
\text { of exposure }\end{array}$} \\
\cline { 3 - 4 } $\begin{array}{l}\text { Culture duration } \\
\text { when aliquot removed }\end{array}$ & $\begin{array}{l}\text { Spectrophotometric } \\
\text { absorbance }\end{array}$ & Test & Control \\
\hline$h$ & $400 \mathrm{~nm}$ & $\mathrm{~Hz}$ & $\mathrm{~Hz}$ \\
& & & \\
6 & 0.017 & 12.4 & $(13.8)$ \\
18 & 0.244 & 8.1 & $(13.5)$ \\
24 & 0.314 & 8.3 & $(13.8)$ \\
36 & 0.412 & 8.7 & $(13.7)$ \\
48 & 0.648 & 4.5 & $(15.2)$ \\
72 & 0.676 & 4.1 & $(15.2)$ \\
96 & 0.704 & Stasis at & $(13.2)$ \\
& & 15 min &
\end{tabular}




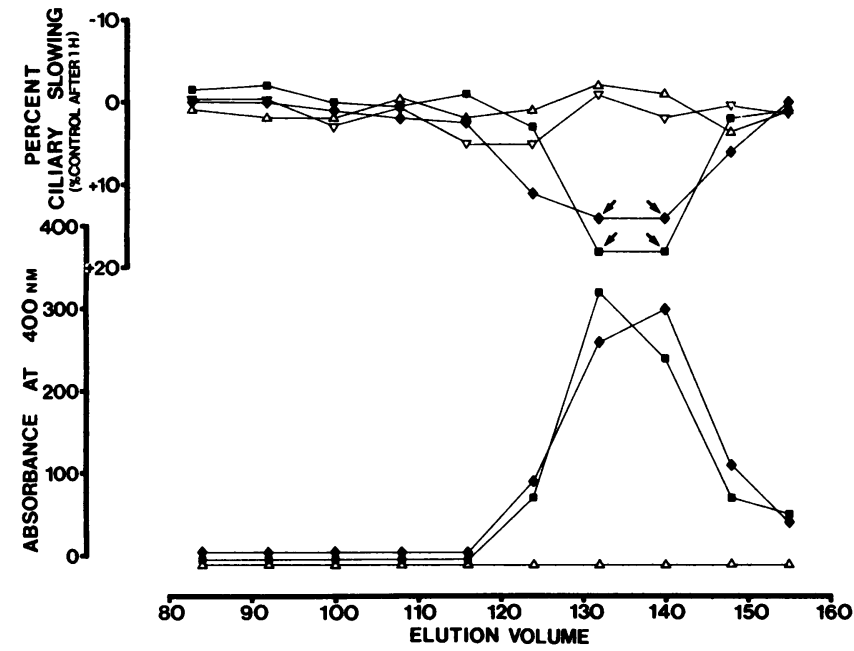

Figure 2. Inhibition of human nasal CBF by eluates of gel-filtered, freeze-dried filtrate; medium $199(\nabla)$, isolate $2(\square)$, isolate $5(\bullet)$, isolate $8(\Delta)$. Absorbance $(400 \mathrm{~nm})$ of filtrates from isolates 2,5 , and $8 .<$, significant $P<0.001 t$ test.

$10 \mu \mathrm{M} 1$-hp caused complete ciliostasis at $240 \mathrm{~min} ; 2 \mu \mathrm{M}$ caused a test value of $7.8 \mathrm{~Hz}$, and a control value of $11.7 \mathrm{~Hz}$ with some dyskinesia and ciliostasis at $210 \mathrm{~min} ; 1 \mu \mathrm{M}$ caused a test value of $8.6 \mathrm{~Hz}$, and a control value of $11.4 \mathrm{~Hz}$ at $150 \mathrm{~min}$; and 0.3 $\mu \mathrm{M}$ caused a test value of $9.6 \mathrm{~Hz}$, and a control value of 11.4 $\mathrm{Hz}$ at $60 \mathrm{~min}$. At this lower $\mathrm{pH}, 1-\mathrm{hp}$ still caused no epithelial disruption and its ciliary-slowing properties were again immediate but tended to progress rather than plateau.

At pH 6.5, $5 \mu \mathrm{M}$ pyocyanin caused a test value of $8.1 \mathrm{~Hz}$, and a control value of $12.2 \mathrm{~Hz}$ with epithelial disruption and ciliostasis at $240 \mathrm{~min}$; and $1 \mu \mathrm{M}$ caused a test value of $8.2 \mathrm{~Hz}$, and a control value of $11.9 \mathrm{~Hz}$ with some epithelial disruption at $240 \mathrm{~min}$.

Measurement of cilioinhibitory factors within culture filtrates. Nine filtrates were assayed for the presence of pyocyanin and 1-hp by HPLC, five of these caused substantial ciliary slowing, two caused minor changes in $\mathrm{CBF}$, and two produced no change in CBF (Table IV). The filtrates from two longer cultures (48 and $96 \mathrm{~h}$ ) were similarly assayed.

The conditions used for HPLC allowed direct analysis of high salt samples without the need for prior extraction (and attendant losses). Pyocyanin eluting at $25.4 \mathrm{ml}$ in the propanol/ acetic acid system was quantitated against an HPLC/UV standard curve. In all the 18-h culture filtrates the bioactivity of the filtrate correlated (Kendall's rank correlation coefficient $\tau=\mathbf{0 . 7 2}$, $P<0.01$ ) with the amount of pyocyanin present, as determined by $A_{280}$ peak height (Table IV). Representative HPLC/UV profiles are shown in Fig. 6.

In each sample in which pyocyanin was observed on HPLC, the characteristic UV spectrum for pyocyanin could be obtained after base extraction of larger quantities of the parent filtrate into chloroform.

1-hp was below the limit of detection in each of the 18-h filtrates examined, although it was detected in the chloroform extract from agar plate cultures.

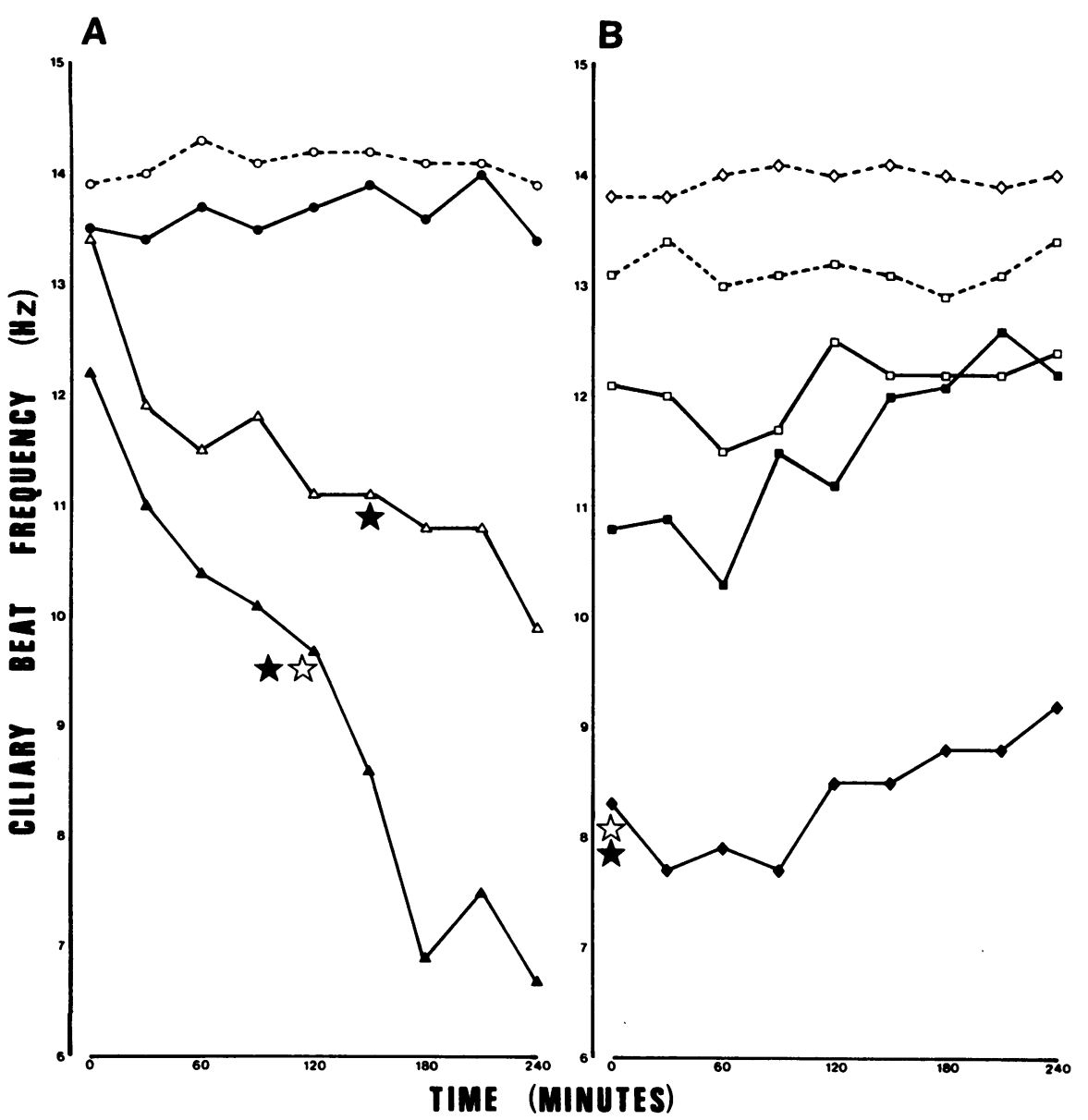

Figure 3. The dose-response effect of purified pyocyanin and purified 1-hp on human nasal CBF at pH 7.4. In $A$, the CBF produced by pyocyanin $10 \mu \mathrm{M}(\bullet), 20 \mu \mathrm{M}(\Delta)$, $100 \mu \mathrm{M}(\Delta)$, and the time-based control $\mathrm{CBF}(0)$ are shown. In $B$, the CBF produced by 1-hp $10 \mu \mathrm{M}(\square), 20 \mu \mathrm{M}(\square)$, and the time-based control CBF ( $\square---\square)$ are shown. Also in $B$ is the CBF produced by $50 \mu \mathrm{M} 1-\mathrm{hp}(\bullet)$ and the time-based control $\operatorname{CBF}(\diamond) . \star=$ ciliary dyskinesia; $\star=$ ciliostasis. 


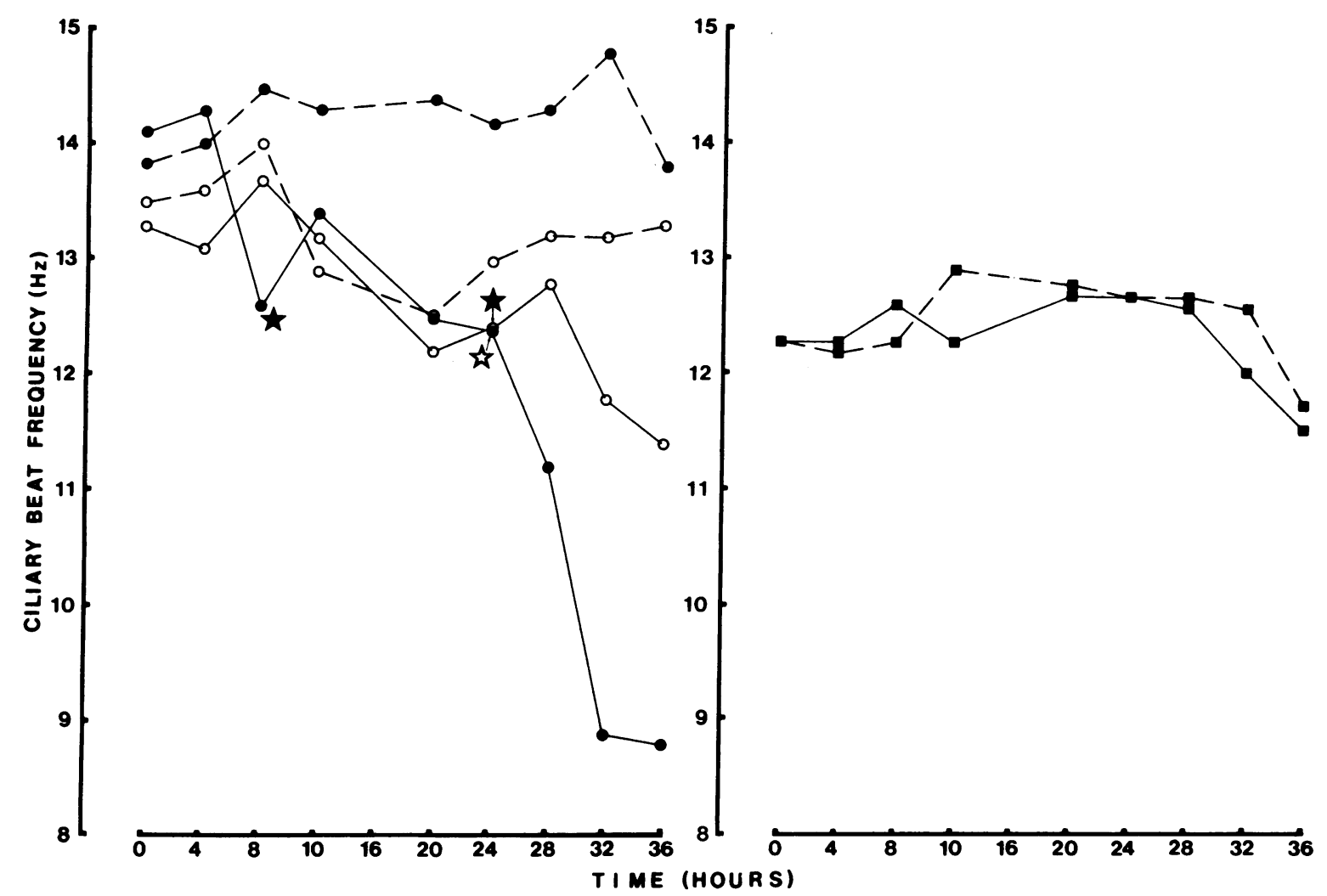

Figure 4. The effect of purified pyocyanin (solid circles, $1 \mu \mathrm{M}$; open circles, $0.1 \mu \mathrm{M}$ ) and 1-hp (solid boxes, $1 \mu \mathrm{M}$ ) on human nasal CBF at pH 7.5 over $36 \mathrm{~h}$; solid lines, test; dashed lines, control. Solid stars, first observation of epithelial disruption; open stars, ciliostasis first observed.

In the filtrates from longer cultures, the pyocyanin content was reduced (Table IV) and other UV-absorbing species became apparent (at 4.3 and $48.5 \mathrm{ml}$ ).

\section{Discussion}

Mucociliary clearance is delayed in patients with chronic bronchial sepsis (17). There are likely to be several reasons for this,

Table IV. Ciliary Slowing Properties and Pyocyanin Concentrations in Active and Inactive Culture Filtrates

\begin{tabular}{lllrl}
\hline Isolate & Duration of culture & Ciliary slowing & Pyocyanin & \\
\hline & $h$ & \% control & $n g / m l$ & $\mu M$ \\
9 & 18 & $71^{*}$ & 4,745 & $(24)$ \\
10 & 18 & $40^{*}$ & 3,297 & $(16)$ \\
11 & 18 & $42^{*}$ & 2,950 & $(15)$ \\
12 & 18 & $31^{*}$ & 1,250 & $(6)$ \\
13 & 18 & $29^{*}$ & 800 & $(4)$ \\
14 & 18 & $15^{*}$ & 0 & \\
15 & 18 & 0 & 0 & \\
16 & 18 & 12 & 0 & \\
17 & 18 & 3 & 0 & \\
13 & 48 & $37^{*}$ & 440 & $(2)$ \\
18 & 96 & $77^{*}$ & 0 & \\
\end{tabular}

$* P<0.001 t$ test, test vs. control at the same experimental time point, 18 d.f. including change in mucus properties and damage to respiratory ciliated epithelium by leucocyte proteolytic enzymes released during the inflammatory response. Human leucocyte elastase has been shown to arrest rabbit ciliary beating in vitro (18), and bronchiectatic sputum sol with elastase activity has been shown to slow the beating of normal human nasal cilia in vitro (19).

Bacterial products may delay clearance by their effect on human cilia. Culture filtrates of $P$. aeruginosa and Haemophilus influenzae slow human nasal ciliary beating in vitro (3). Infection with Mycoplasma pneumoniae destroys human ciliated epithelium (20), and $H$. influenzae causes ciliostasis in rat and chick tracheal organ cultures (21). Southern et al. (22) demonstrated delayed clearance from the lungs of normal mice infected with some strains of $P$. aeruginosa, and this phenomenon appeared to correlate with lecithinase production by the strains. Reimer et al. (23) used rabbit cilia in an in vitro model to investigate filtrates from three strains of $P$. aeruginosa. A concentrationdependent slowing of ciliary beating was demonstrated, and this was reproduced by pyocyanin partially purified by the method of Armstrong et al. (11) (therefore containing 1-hp) and by haemolysin extracted from the organism. Caution must be exercised in extrapolating such results in the rabbit to man, because considerable interspecies variation in susceptibility of cilia to slowing has been demonstrated (24).

$P$. aeruginosa synthesizes numerous products that have been tested in vitro and in vivo $(25,26)$ for possible pathogenic significance. Most clinical isolates of this organism produce a pigment, pyocyanin. Mucociliary clearance is the first-line defense mechanism of the human respiratory tract against inhaled par- 
ticles. Our results show that purified 1-hp, a degradation product of pyocyanin, rapidly slows and disorganizes the beating of cilia and stops a proportion completely, whereas pyocyanin itself slows cilia gradually but finally disrupts ciliary epithelium completely. We have confirmed the potency of these two molecules by synthesis. At lower $\mathrm{pH}$, the bioactivity of both 1-hp and pyocyanin markedly increases. As pH is reduced, there will be increasing protonation of the phenolic hydroxyl group (Fig. 5) which suggests that this may be important for bioactivity-either through enhanced entry into the cell or possibly through more favorable interaction with receptors.

The ciliary-slowing activity of 18 -h culture filtrates correlated ( $r=0.97)$ only with pigment content as measured by spectrophotometric absorbance. Subsequent development of methodology to assay pyocyanin within filtrates showed a similar good correlation $(\tau=0.72)$ between the amount of pyocyanin present and biological activity.

The filtrates of two longer cultures contained less pyocyanin but maintained biological activity. The reduction in amount of pyocyanin is likely to be due to degradation to other phenazine compounds-which may contribute to the ciliary slowing properties of these filtrates from prolonged cultures. Indeed, other less polar UV-absorbing species were observed on HPLC of these filtrates and these may possess cilioinhibitory activity.

We have shown 1-hp to be active and to reproduce the rapid onset of ciliary slowing with subsequent recovery, observed in experiments using some filtrates (Fig. 1). However, there was no evidence of 1-hp in the HPLC profile of culture filtrates and therefore, if present, it is below the limit of detection of this system. It is possible that, if formed from pyocyanin, 1-hp is further metabolized to other (possibly cilioinhibitory) species. 1-hp was however observed in chloroform extracts of agar plate cultures (as noted by Armstrong et al. [11]), perhaps reflecting diffusion of 1-hp away from the immobilized organism. We have recently examined the pulmonary secretions obtained from the lung of a patient suffering from cystic fibrosis removed before heart-lung transplantation and found both pyocyanin and 1-hp to be present. The concentration of each of these pigments was measured using HPLC and found in each case to be in excess of that slowing cilia in vitro.

At the beginning of this century a pigment-containing preparation "pyocyanase", produced from old cultures of $P$. pyocyanea, was shown to have antibacterial properties and was used in the treatment of patients (27). During the first World War, physicians noted the improvement in erysipelas when $P$. pyocyanea infection supervened (27). In 1947, Young (28) described a number of pigments produced by $P$. aeruginosa, including
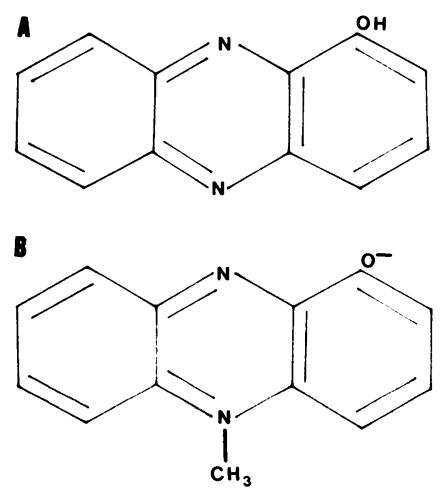

Figure 5. The molecular structure of 1-hp $(A)$ and pyocyanin $(B)$.

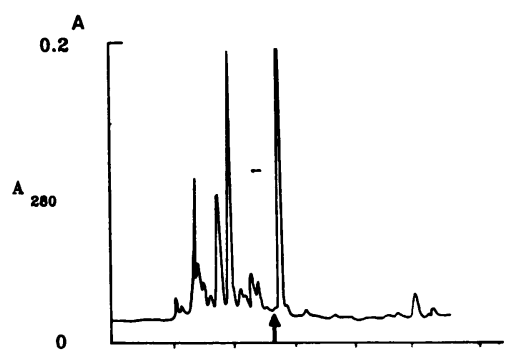

Figure 6. Representative HPLC UV absorbance profiles from the purification of culture filtrates. The samples were chromatographed directly (system iii, propan-2-ol/acetic acid) without prior purification. The elution position of pyocyanin is marked with an arrow. (A) $\mathrm{A}_{280} \mathrm{UV}$ profile of an inactive filtrate. $A$ number of UV-absorbing peaks are present but there is no evidence of pyocyanin or 1-hp. (B) $\mathrm{A}_{280}$ UV profile of an active filtrate showing the presence of pyocyanin.

pyocyanin, which is blue and its yellow breakdown product 1hp. Schoental (29) isolated three antibacterial substances from chloroform extracts of $P$. pyocyanea, two of which were pyocyanin and 1-hp.

Pyocyanin is a redox dye (30), and its complicated mass spectral behaviour (notably on thermospray LC/MS but also under d.e.i. ionization) arises from its unusual structural properties (i.e., its zwitterionic nature, which confers solubility in both water and chloroform). Pigment-containing preparations from $P$. aeruginosa have been shown to impair cellular respiration (11). Oxygen consumption by mouse liver mitochondria was measured for $8 \mathrm{~min}$ after addition of the potential inhibitor. The active fraction that caused inhibition of cellular respiration was 1-hp while pyocyanin was inactive (11). The apparent discrepancy between our results and those of Armstrong et al. (11) may arise from the slower onset of pyocyanin action.

Early in an infection it would be a critical advantage for pseudomonas to produce a virulence factor that compromises ciliary function and enables the organism to establish itself within the respiratory tract. We propose that, in the microenvironment of the ciliated epithelium, pseudomonas produces pyocyanin and 1-hp, which paralyze mucociliary clearance. Both are active in vitro in nanomolar concentrations. Pyocyanin slows ciliary beating and ultimately disrupts the epithelium completely. 1$\mathrm{hp}$, as well as slowing ciliary beating, also disorganizes the beating pattern. Once established, subsequent spread of the organism within the lung would be facilitated by these properties, leading to considerable morbidity and mortality in patients with cystic fibrosis and other forms of severe bronchiectasis.

\section{Acknowledgments}

Dr. MacDermot is a Wellcome Senior Research Fellow. Aspects of this work were supported by a Medical Research Council Programme Grant and the National Fund for Research into Crippling Diseases.

\section{References}

1. Hoiby, N. 1982. Microbiology of lung infections in cystic fibrosis patients. Acta Paediatr. Scand. 301(Suppl.):33-54. 
2. Afzelius, B. A., and B. Mossberg. 1980. Immotile cilia. Thorax. 35:401-404.

3. Wilson, R., D. Roberts, and P. Cole. 1985. Effect of bacterial products on human ciliary function in vitro. Thorax. 40:124-131.

4. Rutland, J., and P. J. Cole. 1980. Non-invasive sampling of nasal cilia for measurement of beat frequency and study of ultrastructure. Lancet. ii:564-565.

5. Greenstone, M., R. Logan-Sinclair, and P. J. Cole. 1984. An automated method of reading ciliary beat frequency. IRCS Med. Sci. 12: 715-716.

6. Sierra, G. 1957. A simple method for the detection of lipolytic activity of microorganisms and some observations on the influence of the contact between cells and fatty substances. Antonie Leeuwenhoek $J$. Microbiol. 23:15-22.

7. Jeffries, C. D., D. F. Hoffman, and D. G. Guse. 1957. Rapid method for determining the activity of microorganisms on nucleic acids. J. Bact. 73:590-591.

8. Cowan, S. T., and K. J. Steel. 1974. Manual for the identification of medical bacteria. Cambridge University Press, Cambridge. 37.

9. Chester, I. R., P. M. Meadow, and T. L. Pitt. 1973. The relationship between the O-antigenic lipopolysaccharides and serological specificity in strains of Pseudomonas aeruginosa of different O-serotypes. J. Gen. Microbiol. 78:305-318.

10. Pitt, T. L., and L. C. Raisbeck. 1978. Degradation of the mucoid polysaccharide of Pseudomonas aeruginosa by Beneckea pelagia. J. Appl. Bacteriol. 45:297-300.

11. Armstrong, A. V., D. G. S. Stewart Tull, and J. S. Roberts. 1971. Characterisation of the Pseudomonas aeruginosa factor that inhibits mouse-liver mitochondrial respiration. J. Med. Microbiol. 4:249-262.

12. King, E. O., M. K. Ward, and D. E. Raney. 1954. Two simple media for the demonstration of pyocyanin and fluorescin. J. Lab. Clin. Med. 44:301-307.

13. Morris, H. R., A. T. Etienne, A. Dell, and R. Alberquerque. 1980. A rapid and specific method for the high resolution purification and characterisation of neuropeptides. J. Neurochem. 34:574-582.

14. Corbett, J. F. 1964. The infra-red and ultra-violet spectra of hydroxyphenazines. Spectrochim. Acta. 20:1665-1675.

15. Holliman, F. G., R. A. W. Johnstone, and B. J. Millard. 1967. Aspects of mass spectra of organic compounds. Part V. Some phenazines. J. Chem. Soc. (Lond.). 2351-2356.

16. Luk, C. K., and M. Dulfano. 1983. Effect of $\mathrm{pH}$, viscosity and ionic strength changes on ciliary beating frequency of human bronchial explants. Clin. Sci. 64:449-451.

17. Lourenco, R. V., R. Loddenkemper, and R. W. Carton. 1972. Patterns of distribution and clearance of aerosols in patients with bronchiectasis. Am. Rev. Respir. Dis. 106:857-866.

18. Tegner, H., K. Ohlsson, W. G. Toremalm, and C. von Mecklenburg. 1979. Effect of human leucocyte enzymes on tracheal mucosa and its mucociliary activity. Rhinology. (Rott.). 17:199-206.

19. Smallman, L. A., S. L. Hill, and R. A. Stockley. 1984. Reduction of ciliary beat frequencies in vitro by sputum of patients with bronchiectasis: a serine proteinase effect. Thorax. 39:663-667.

20. Collier, A. M., and W. A. Clyde. 1977. Relationships between Mycoplasma pneumoniae and human respiratory epithelium. Infect. Immun. 3:694-701.

21. Denny, F. 1974. Effect of a toxin produced by Haemophilus influenzae on ciliated respiratory epithelium. J. Infect. Dis. 129:93-100.

22. Southern, P. M., B. B. Mays, A. K. Pierce, and J. P. Sanford. 1970. Pulmonary clearance of Pseudomonas aeruginosa. J. Lab. Clin. Med. 76:548-559.

23. Reimer, A., K. Klementsson, J. Ursing, and B. Wretling. 1980. The mucociliary activity of the respiratory tract; 1 . Inhibitory effects of products of Pseudomonas aeruginosa on rabbit trachea in vitro. Acta Otolaryngol. 90:462-469.

24. Rutland, J., A. Penketh, W. M. Griffin, M. E. Hodson, J. C. Batten, and P. J. Cole. 1983. Cystic fibrosis serum does not inhibit human ciliary beat frequency. Am. Rev. Respir. Dis. 128:1030-1034.

25. Liu, P. V. 1974. Extracellular toxins of Pseudomonas aeruginosa. J. Infect. Dis. 130(Suppl.):94-99.

26. Cash, H. A., D. C. Straus, and J. A. Bass. 1983. Pseudomonas aeruginosa exoproducts as pulmonary virulence factors. Can. J. Microbiol. 29:448-456.

27. Florey, H. W., E. Chain, W. G. Heatley, M. A. Jennings, A. G. Sanders, E. P. Abraham, and M. E. Florey. 1949. Antibiotics. Vol. 1. Oxford University Press, Oxford. 20-26.

28. Young, G. 1947. Pigment production and antibiotic activity in cultures of Pseudomonas aeruginosa. J. Bacteriol. 54:109-117.

29. Schoental, R. 1941. The nature of the antibacterial agents present in Pseudomonas Pyocyanea cultures. Brit. J. Exp. Path. 22:137-147.

30. Hewitt, L. F. 1936. Oxidation: reduction potentials in Bacteriology and Biochemistry. 6th Ed. Livingstone, Edinburgh. 64-66. 